\title{
Integrated Modeling Methodologies and Languages
}

\author{
Sabah Al-Fedaghi \\ Computer Engineering Department, Kuwait University \\ P.O. Box 5969 Safat 13060, Kuwait \\ $+96599939594$ \\ sabah.alfedaghi@ku.edu.kw
}

\author{
Haya Alahmad \\ IT Department, Ministry of Public Works, South Surra, \\ Kuwait \\ haya.alahmad@ieee.org
}

\begin{abstract}
Two ${ }^{1}$ state-of-the-art languages being used in the field of integrated modeling and management of systems are $\mathrm{OMG}$ Systems Modeling Language (SysML) and Object-Process Methodology (OPM). SysML is based on UML 2, a de-facto standard in software engineering. OPM is chartered as ISO/PAS 19450 for system and process modeling. Aspects of these two diverse modeling methodologies have been comparatively analyzed and examined. This paper points to yet another approach that takes a methodology oriented to flow of things to provide an underlying conceptual model on which to construct a fundamental representation of the information or physical system. Adopting a similar analysis technique of comparing SysML and OPM, we examine features of a flow of things orientation based on a concrete sample problem. To demonstrate the feasibility of this methodology in a real environment, we present a case study of an actual IT department of a government ministry, and the results point to the viability of the approach as a foundation for modeling and management of systems.
\end{abstract}

\section{CCS CONCEPTS}

- Modeling and simulation $\rightarrow$ Model development and analysis $\rightarrow$ Modeling methodologies

\section{KEYWORDS}

Process; object; behavior; conceptual modeling; OPM; UML; user modeling

\section{ACM Reference format:}

S. Al-Fedaghi and H. Alahmad. 2018. Integrated Modeling Methodologies and Languages. In Proceedings of International Conference on Ubiquitous Information Management and Communication, Langkawi, Malaysia, January 2018, (IMCOM '18), 8 pages. https://doi.org/10.1145/3164541.3164605

Permission to make digital or hard copies of part or all of this work for personal or classroom use is granted without fee provided that copies are not made or distributed for profit or commercial advantage and that copies bear this notice and the full citation on the first page. Copyrights for third-party components of this work must be honored. For all other uses, contact the owner/author(s).

IMCOM '18, January 5-7, 2018, Langkawi, Malaysia

(C) 2018 Copyright is held by the owner/author(s).

ACM ISBN 978-1-4503-6385-3/18/01.

https://doi.org/10.1145/3164541.3164605

\section{INTRODUCTION}

System development has become a challenging task for large and complex systems, resulting in abandonment of traditionally document-centric system development approaches. Current solutions to reduce complexity include adopting of model-based systems engineering, resulting in significant progress with the introduction of such methodologies as object-oriented modeling [1-3] and object-process methodology [4-5]. Some of these approaches emphasize combining of structure and behavior to reduce complexity, e.g., [6-7]. Stress is also placed on conceptual models [8] that can be used to facilitate user understanding of diagrammatic description [4].

One of the main goals of conceptual modeling is to improve system analysis and enable its architecting and detailed design. Early model construction provides for verification and validation of a system prior to implementation and integration of its components. Other benefits of a conceptual model include improving communication between stakeholders and enabling the reuse of some of its components. [9]

Typically, a conceptual model provides a language for representing a system; two state-of-the-art representative languages are OMG Systems Modeling Language (SysML) [1011OMG 2007a-b] and Object-Process Methodology (OPM) [4]. Grobshtein and Dori [9] examined aspects of these two diverse modeling languages and note that "The evaluation was done on the basis of a concrete sample problem, in which multiple aspects of the system were modeled in both SysML and OPM" [9]. SysML is based on UML 2, a de-facto standard in software engineering, with several extensions for general systems engineering. OPM is chartered as ISO/PAS 19450 for system and process modeling (ISO/PAS 19450:2015). OPM is based on a single diagram type, whereas SysML has inherited the model multiplicity of UML. In OPM, the entire system model is based on a hierarchy of diagrams [9].

This paper points to another methodology for modeling systems that takes a completely different approach from objectorientation and object-process orientation. It is a methodology oriented to flow of things that can provide an underlying conceptual model on which to construct a fundamental representation of the basic system. Adopting Grobshtein and Dori's [9] method of analysis based on a concrete sample problem to examine multiple aspects of a system in both SysML and OPM, 
this paper takes the sample problem and models it using a floworiented approach.

To demonstrate the feasibility of the proposed methodology in a real environment, a case study of an actual IT department of a government ministry is presented; the results point to the viability of the approach as a foundation for common understanding of systems.

For the sake of a self-contained paper, section 2 briefly describes the conceptual model, called the Flowthing Model (FM), on which the flow-based representation is built. FM has been utilized in many applications [11-13]. Section 3 introduces a complete example that illustrates the static and dynamic aspects of the model. Section 4 applies the concrete sample problem used to compare SysML and OPM to the proposed methodology. Section 5 describes the case study.

\section{FLOWTHING MACHINE}

The world is complex and includes objects, substances, actions, events, ... as just a few of its things. The Flowthing Machine (FM) views these things in terms of flows that exist in diverse fields. The basic construct in FM is a thing, existing within a diagrammatic (abstract) machine (see Figure 1).

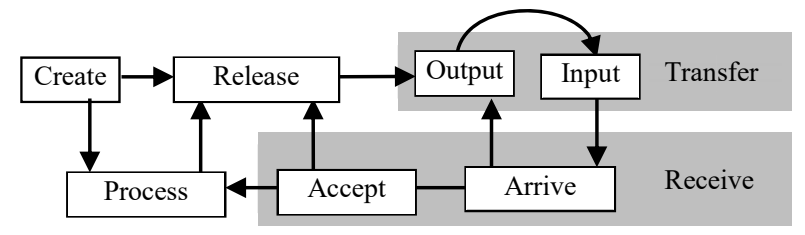

Figure 1: Flow machine.

FM represents a process (not to be confused with the process stage shown in Figure 1). ISO 9000:2005 defines a process as "transformation of media of inputs into outputs." Such a definition is incomplete, because processes may create results and not necessarily by transforming inputs. In FM, things represent a range of physical and abstract items, data, information, signals, objects, events, etc. As Figure 1 shows, FM is based on six stages (states), as follows:

- Arrive: A thing reaches a new flow machine (e.g., data arrive at a buffer in a router machine).

- Accepted: A thing is permitted to enter a flow machine (e.g., correct address for a delivery); if arriving things are also always accepted, Arrive and Accept can be combined as a Received stage.

- Released: A thing is marked as ready to be transferred outside the flow machine (e.g., in an airport, airline passengers with cleared passports waiting to board).

Note that not every incoming transferred thing is accepted and not every released thing is output (transferred). Things may be returned upon arrival, e.g., having a wrong address. Similarly, released things may not be transferred, e.g., a channel is down.

- Processed (changed): The flowthing goes through some kind of transformation that changes its form but not its identity, e.g., a message translated into another language or a number changed from decimal to binary.

- Created: A new flowthing is born (created) in a flow machine (e.g., a data mining program generates a conclusion).

- Transferred: The flowthing is transported somewhere from/to outside the flow machine (e.g., packets reaching ports in a router, but still not in the arrival buffer). Note that typically a process is described with the essential components of input and output.

In general, a flow machine is conceived as an abstract machine that receives, processes, creates, releases, and transfers flowthings. The stages in this machine are mutually exclusive (i.e., a flowthing in the process stage cannot be in the create stage or the release stage at the same time). An additional stage of Storage can also be added to any machine to represent the storage of flowthings; however, storage is not an exclusive stage because there can be stored processed flow things, stored created flowthings, etc.

A flowthing is defined as a thing that can be created, released, transferred, received, or processed while flowing within and between machines. Hereafter, a thing means a flow thing.

FM also uses the following notions:

- Spheres and subspheres: These are the environments of the machine. Multiple machines can exist in a sphere if needed. A sphere can be an entity (e.g., a company, a customer), a location (a laboratory, a waiting room), a communication media (a channel, a wire). A flow machine is a subsphere that embodies the flow; it itself has no subspheres.

- Triggering: Triggering is the activation (denoted in FM diagrams by a dashed arrow) of a flow. It is a (causative) dependency among flows and parts of flows. A flow is said to be triggered if it is created or activated by other flows (e.g., a flow of electricity triggers a flow of heat), or activated by another point in the flow.

\section{EXAMPLE}

Grobshtein and Dori [9] evaluated, compared, and analyzed the two modeling languages SysML and OPM by investigating a sample modeling problem given in SysML specification [10], the Sport Utility Vehicle (SUV) system. The system was modeled using SysML diagrams [10] and their semantic equivalent OPDs [9]. Figure 2 depicts goal level use cases associated with "Operate the Vehicle." Figure 3 describe the OPM system diagram. The top-level diagram includes several objects, as well as one process, the main function of the system, to be decomposed in lower-level OPDs.

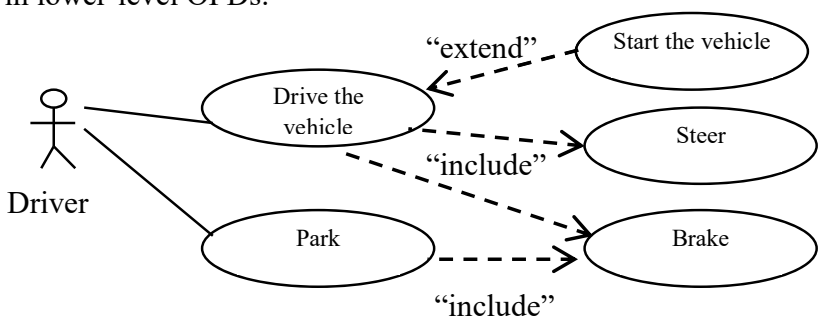

Figure 2: Use case diagram associated with "Operate the Vehicle" in SysML (redrawn, partial from [10]). 


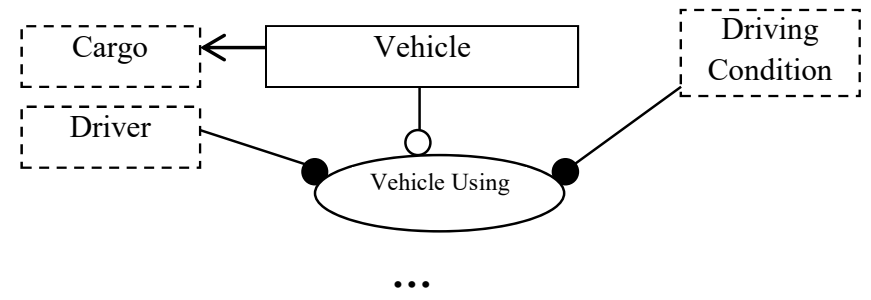

Figure 3: System Diagram of the SUV system in OPM (redrawn, partial from [9]).

In Figure 4, an OPM diagram is created by zooming the "Vehicle Using" process from the top-level system diagram, exposing its sub-processes.

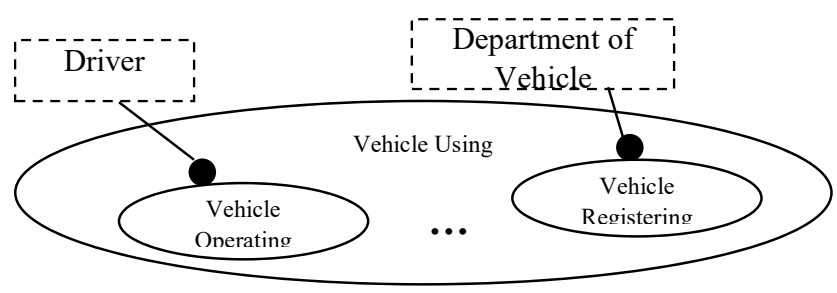

Figure 4: Zoom of the vehicle using process in OPM (redrawn, partial from [9]).

Our purpose here is not to give a fair description of this modeling methodology; rather the goal is to illustrate its general thinking style and the diagramming language it uses. In FM the thinking style is based on the flow machine described previously. It starts by identifying a thing and discovering its flow to its end. Take for example a vehicle. The physical vehicle itself flows in various ways:
- It is processed by the driver

- It flows onto roads, in different weather conditions and in relation to external things

- It flows to a destination

- It and its engine, electrical power system, and brake system "flow" through various states

- Its movement is created and processed

Accordingly, a first step in creating an FM model of a vehicle system is shown in Figure 5, where things and their flows are roughly indicated. The main sphere is the vehicle (circle 1 in the figure),

- $\quad$ as a physical thing (2) that flows to roads, a park, ...

- receiving several diverse flows: registration certificate, cargo, insurance, ... (3)

- $\quad$ with internal flows of braking, state, movement, ... (4)

The other main sphere is the driver (5):

- $\quad$ as a physical thing that flows to the vehicle (6)

- $\quad$ and activates behavior of the vehicle (7) - in this model, as we will show, behavior is a flow of events.

Note that such flow-based thinking is uniform in using only the notions of spheres (e.g., classes in object-oriented terminology), things, and flows, including triggering. Also, because of space limitations and without loss of generality, we model only certain operational aspects of a vehicle.

\subsection{Static FM Description}

Figure 6 shows a more complete version of the static description of the example. It can be described as follows:

The (physical) driver (1) enters the vehicle (2), where he/she can trigger any of the processes listed (3). The driver is expected to start by turning a key (4) which triggers (5) the vehicle to be in the state ON (6), activating the engine (7) and brake (8) systems.

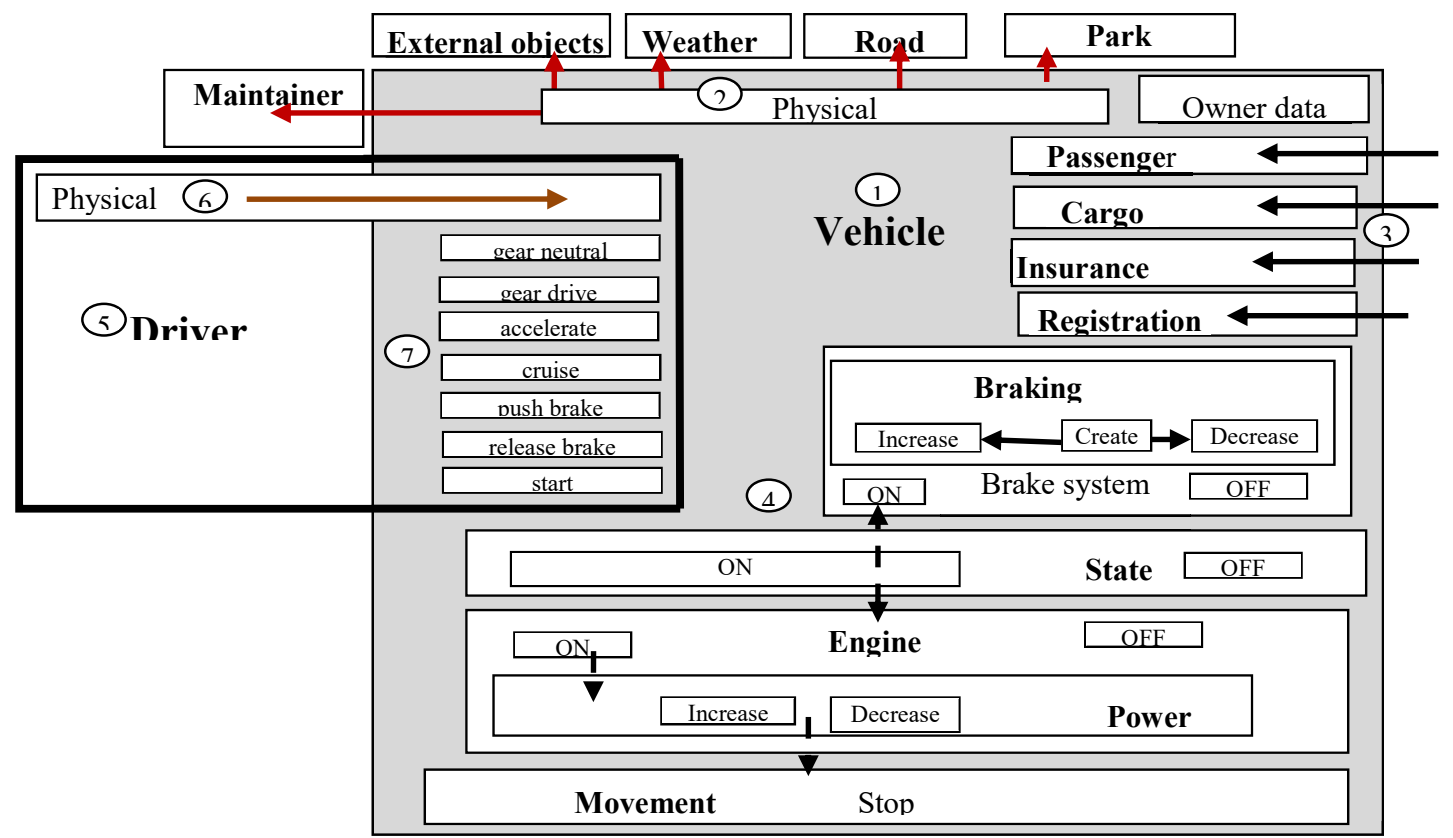

Figure 5: A first general FM description of the example of the vehicle. 


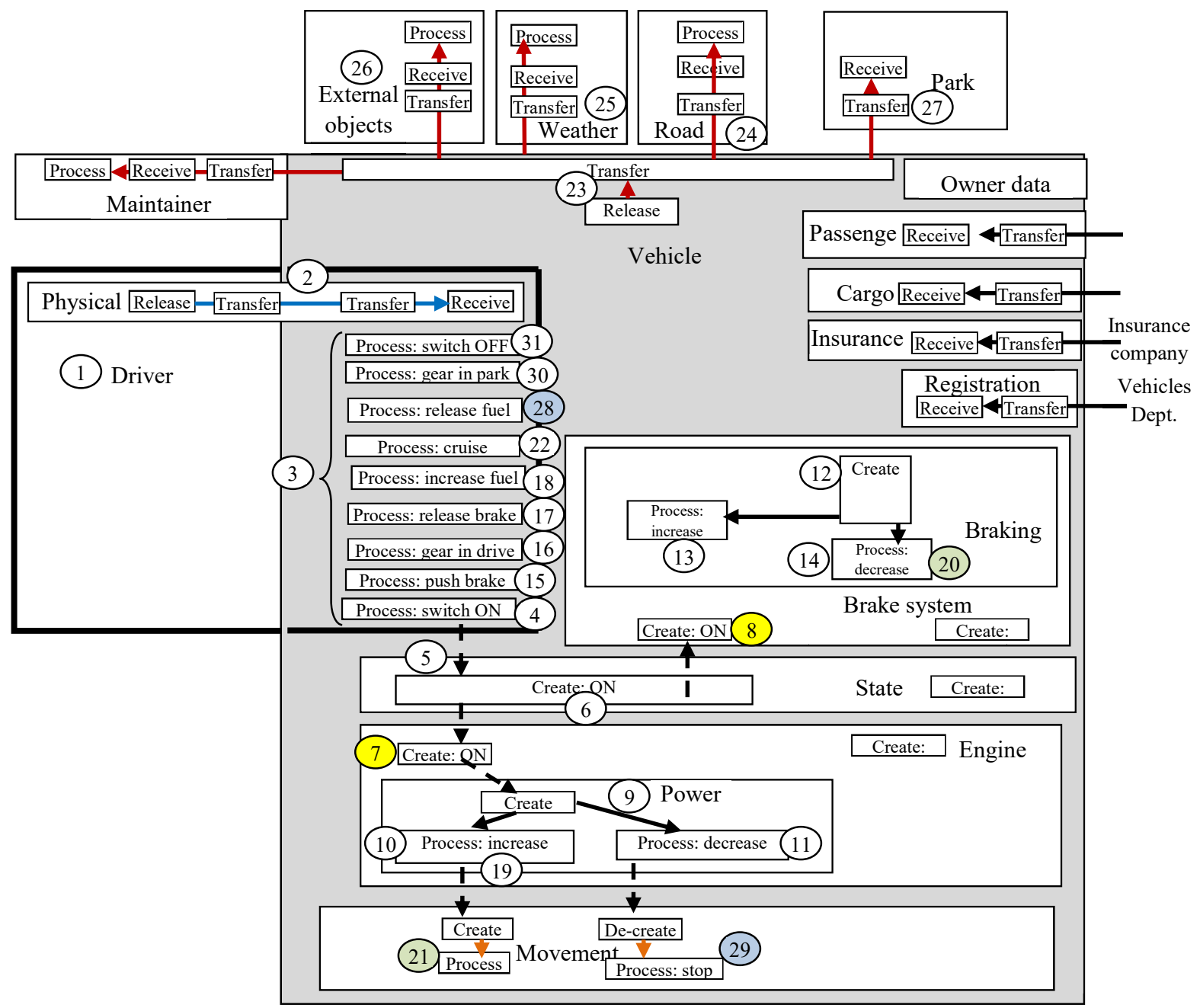

Figure 6: FM description of the example of the vehicle with emphasis on operations.

Turning ON the engine creates power available to be used (9) that is coordinated with the brakes in moving the car at different levels: increasing power (10) or decreasing it (11). Also, turning ON the brake system (8) provides the ability to create (12) and either increase (13) or decrease (14) friction to control the vehicle's momentum,

Under this condition, the driver usually pushes the brake pedal (15) while putting the gear in drive (16). Accordingly, the driver releases the brake (17) and pushes the accelerator to give it fuel (18), causing an increase in engine power (19) and decrease in braking (20), thus causing the vehicle to move (21).

The driver steers (22) the (physical) vehicle on roads (23-24), within a weather sphere (25) and among external objects (26) to a park (27). In the park, the driver pushes the brake pedal (15) and releases the accelerator (28), thus decreasing (11) the power and stopping the vehicle's movement (29). Finally, the driver puts the gear in park (30) and turns the switch OFF (31).

A similar "flow tracking" can be applied to other aspects of the vehicle by input of data about owner, insurance, registration, and the flow of passengers and cargo.

\subsection{System Behavior}

After specifying the main use cases in SysML and the system diagram of the SUV system in OPM, Grobshtein and Dori [9] further model and analyze top-level behavior. SysML diagrams include the sequence and state diagrams, and OPM modeling at this level includes zooming in on the driving process (Figure 7). "The Vehicle Starting subprocess occurs if the operational status of the vehicle is off. As a result of this subprocess occurrence, the vehicle's operational status is changed to operate. This diagram demonstrates for the first time the combination of objects, processes, and states in a single diagram." [9]

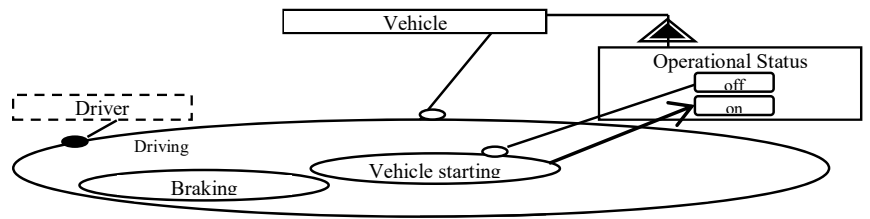

Figure 7. Zoomed-in process (redrawn, partial from [9]) 
In FM, modeling of behavior takes place in a phase that occurs after the static description is created (e.g., Figure 6) and involves modeling the "events space," where an event takes place or happens. An event is specified by its spatial area or subgraph, its time, the event's own stages, and possibly by other descriptors such as intensity or extent (strength).

Note that a conceptual event refers to sets of (elementary) events extended in space and time that, in the context of the involved model, together form a meaningful event. Events appear at different levels, e.g., creating a thing is an event in itself; however, modeling focuses on "meaningful" events. Not every event in history is historically meaningful.

Figure 8 shows the event: the maintainer receives the vehicle. It includes time of event, the occurrence of the event and its duration, and the "event space" as a subdiagram of the static description in Figure 6.

To simplify the drawings of events, here we will not draw the time and stages of the events but focus on their spaces. We can select the following 11 events of interest in the vehicle system shown in Figure 9:

V1: Driver enters the car

V2: Driver starts the car (changes to ON state of the vehicle, engine, and brake systems).

V3: Driver pushes the brake pedal

V4: Driver puts the gear in drive (power is now available)
V5: Driver releases the brake

V6: Driver pushes the fuel pedal

V7: The car moves

V8: Cruising: the vehicle moves on roads in conditions of weather and external circumstances

V9: The car enters the park

V10: Driver releases the fuel pedal

V3: Driver pushes on brake pedal

V11: Vehicle stops movement

V12: Driver changes the gear to park

V5: Driver releases the brake

V11: Driver turns the switch OFF

Accordingly, a control for the system can be developed by identifying the permitted chronology of events, as shown in Figure 10. The dashes between some events indicate parallelism.

Event: the maintainer receives the vehicle

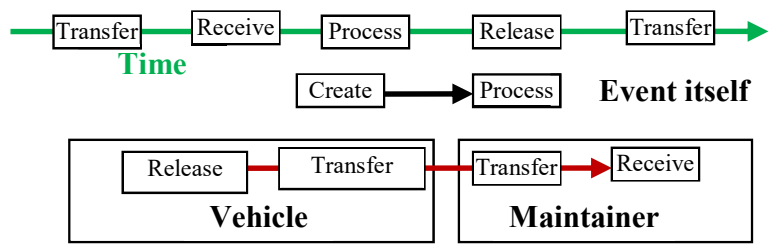

Figure 8. Sample event.

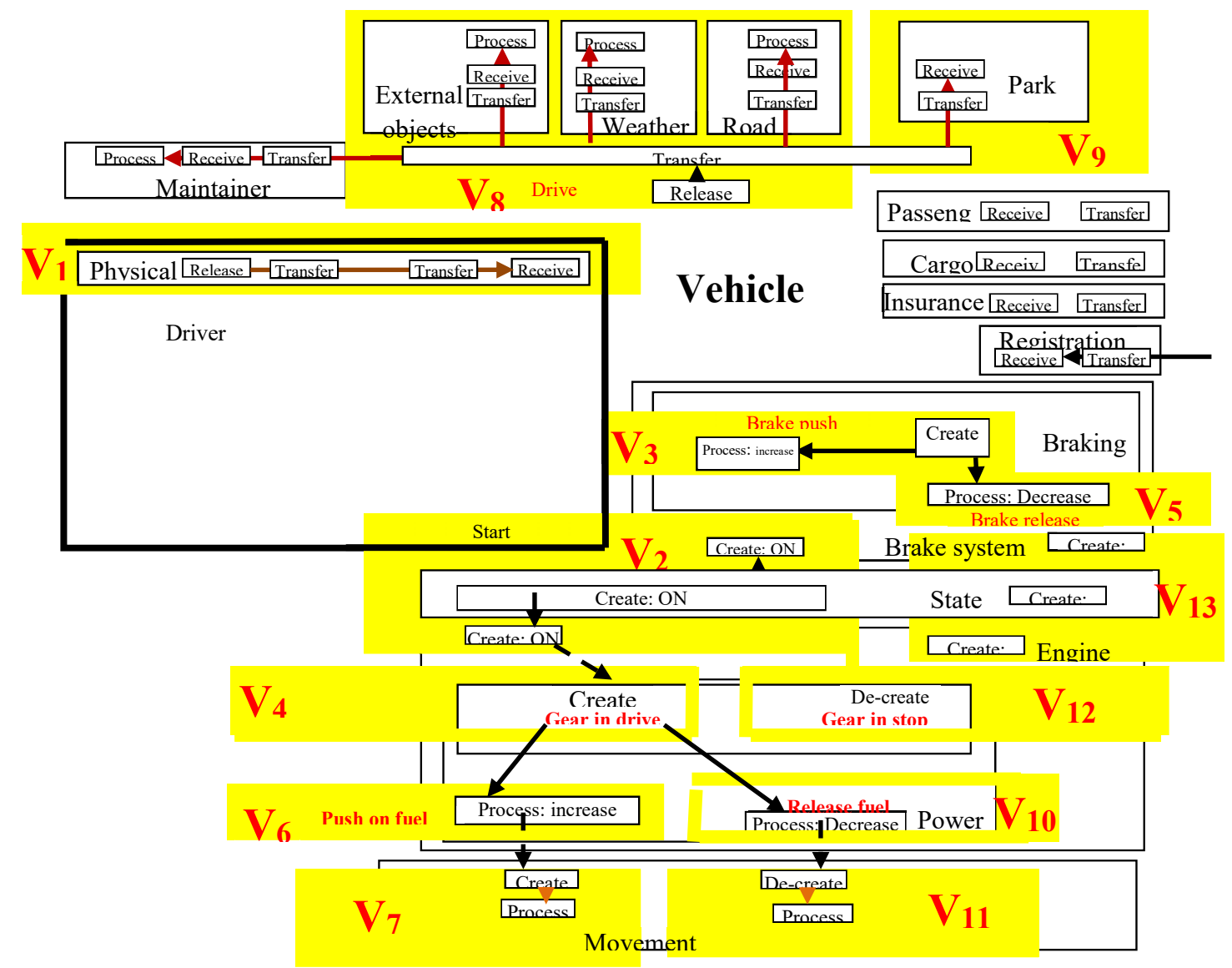

Figure 9: Events in example of the vehicle. 


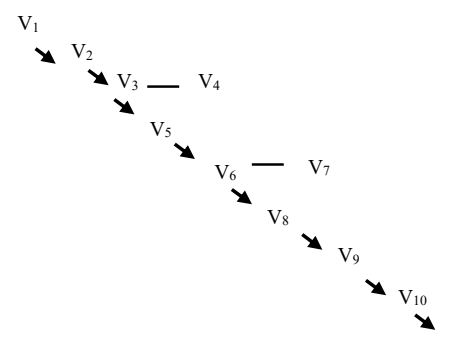

Figure 10: Chronology of events in cruising to the park.

\section{MODEL IMPLEMENTATION}

While the previous section gives a glimpse into FM principles and techniques, this section strengthens validation of FM as a viable method by applying it to the procedures of an actual IT department of a government ministry as a case study. Here, observations are limited to IT processes management, a kind of service desk within the discipline of IT service management.

The case study is a description of a particular operation in the IT department of the Ministry of Public Works, Kuwait. The network team in the ministry administers all aspects of a network that connects the data center to its clients (in the IT sense). The offered services provide use of certain servers, including email server and file server, to access the Internet and external and internal applications.

\subsection{Email Control Process}

In the flow model shown in Figure 11, we address the case of email service in a number of scenarios.

\subsubsection{Logging-In Action}

First, a user (1 in the figure) must enter an email address (2) and password (3); the email system of the client sender (4) then sends a request (5) to the MBX (mailbox email server, 6 - lower right corner) to check for permission. The request (7) to the MBX flows as follows:

- $\quad$ First through switches and server farm (8)

- $\operatorname{MBX}(9)$ then authenticates the user and sends the request (10) to the Hub\&Cas server to apply policies that fit the user in the Hub\&Cas server (11).

- The Hub\&Cas (12) returns it to the MBX server; the MBX server then takes the required data (13) to display the home page of the email system to that user and sends it back through the server farm and the switches (14) and finally to the client (15).

\subsubsection{Email Retrieval}

After logging in, the user might want to open an email shown in a list. When he or she clicks on that email. the request (16) again goes through the switches to the server farm, and the server farm redirects the request to the $\mathrm{MBX}$ server.

- The MBX (17) processes the request to retrieve the requested data and display forums to the user.

- $\quad \operatorname{MBX}(18)$ forms the requested email package and sends it to the requester through the server farm (8) and switches (14) until it reaches the client for display (19).

\subsubsection{Sending Email}

To compose an email (20), the user clicks on the Compose icon. The email system reacts by displaying a form in which the user can enter the content of an email (21).

- The user starts typing the email (22), including an address (23).

- After finishing, the user clicks Send (24) to trigger a request for flow of the email.

- The email send-request flows through the switches to the server farm (8) until it reaches the server Hub\&Cas (25) to apply rules and attach to the email.

- Then, the server resends it (26) to a server called Symantec Messaging Gateway (SMG, 27) that protects against spam and malware. The SMG scans the email and then sends it to the MBX server.

- $\quad$ The MBX saves a copy of the contained email (28) and sends it through the server farm and the switches until it reaches the receiver client (29).

- A reply (if any) can be created (30) and would flow (31) exactly the same as the flow of the sent email just described until it is displayed (32) to the Client 1/ Email system (4).

- The email send-request flows through the switches to the server farm (8) until it reaches the server Hub\&Cas (25) to apply rules and attach to the email.

- Then, the server resends it (26) to a server called Symantec Messaging Gateway (SMG, 27) that protects against spam and malware. The SMG scans the email and then sends it to the MBX server.

- $\quad$ The MBX saves a copy of the contained email (28) and sends it through the server farm and the switches until it reaches the receiver client (29).

- A reply (if any) can be created (30) and would flow (31) exactly the same as the flow of the sent email just described until it is displayed (32) to the Client 1/ Email system (4).

\section{CONCLUSIONS}

This paper introduces a flow-based modeling framework, FM, as another approach to modeling of systems and demonstrates its capabilities by modeling portions of an actual system. A weakness of FM is lack of a computer-based design support system in this phase of development because of deficiencies in the current research environment (e.g., no $\mathrm{PhD}$ program). Thus, an aim of this paper is to attract the attention of researchers to the potential capabilities of this approach. 


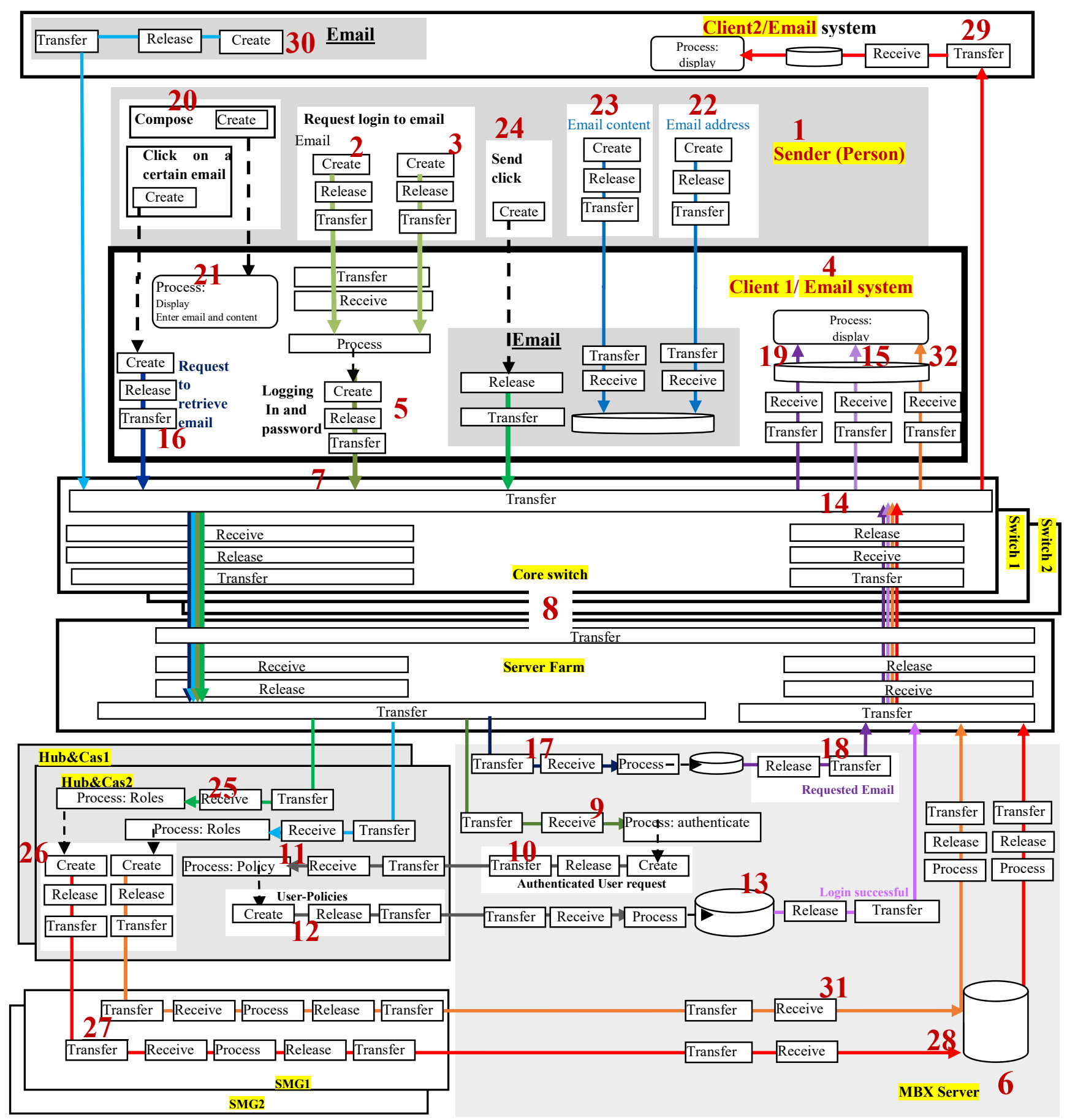

Figure 11: Email process flow. 


\section{REFERENCES}

[1] Coad, P. and Yourdon, E. 1991. Object-Oriented Design. Youdon Press, Englewood Cliffs, NJ, USA.

[2] Jacobson, I. 1991. Object-Oriented Software Engineering. ACM Press, New York, USA.

[3] Rumbaugh, J., Blaha, M., Premerlani, W., Eddy, F., and Lorensen, W. 1991. Object-Oriented Modeling and Design. Prentice-Hall, Upper Saddle River, NJ, USA.

[4] Dori, D. 2002. Object-Process Methodology: A Holistic Systems Paradigm. Springer, Berlin.

[5] Dori, D. 2011. Modeling Knowledge with Object-Process Methodology. http://esml.iem.technion.ac.il/wp-content/uploads/2011/08/Object-ProcessMethodology.pdf

[6] Dori, D. 2001. Object-process methodology applied to modeling credit card transactions. J. Database Manage., 12(1), 4-14

[7] Shoval, P. and Kabeli, J. 2001. FOOM: functional and object-oriented analysis and design of information systems: an integrated methodology. J. Database Manage., 12(1), 15-25
[8] Schichl, H. 2004. Models and history of modeling. In Modeling Languages in Mathematical Optimization, J. Kallrath, Ed. Kluwer Academic, 25-36.

[9] Grobshtein, Y. and Dori, D. 2008. Evaluating aspects of systems modeling languages by example: SysML and OPM. In Proceedings, INCOSE 18th Annual International Symposium, 6th Biennial European Systems Engineering Conference (Utrecht, The Netherlands, June 15-19, 2008).

[10] OMG. 2007. OMG Systems Modeling Language Specification V1.0 (OMG document number formal/2007-09-01).

[11] Al-Fedaghi, S. and Alahmad, H. 2017. Orientation in conceptual modeling frameworks. Paper presented at 3rd IEEE International Conference on Big Data Intelligence and Computing (Orlando, FL, USA, November 6-10, 2017).

[12] Al-Fedaghi, S. 2016. Activity recognition and sensor positioning. Paper presented at IEEE International Conference on Systems, Man, an Cybernetics (IEEE SMC 2016) (Budapest, Hungary, October 9-12, 2016).

[13] Al-Fedaghi, S. 2007. How sensitive is your personal information? Pape presented at 22nd ACM Symposium on Applied Computing (ACM SAC 2007) (Seoul, Korea, March 11-15, 2007). 